\title{
Peptide receptor radionuclide therapy as a potential tool for neoadjuvant therapy in patients with inoperable neuroendocrine tumours (NETs)
}

\author{
Anna Sowa-Staszczak • Dorota Pach • Robert Chrzan • Malgorzata Trofimiuk • \\ Agnieszka Stefańska • Monika Tomaszuk • Maciej Kołodziej • Renata Mikołajczak • \\ Dariusz Pawlak • Alicja Hubalewska-Dydejczyk
}

Received: 12 January 2011 / Accepted: 14 April 2011 /Published online: 11 May 2011

(C) The Author(s) 2011. This article is published with open access at Springerlink.com

\begin{abstract}
Purpose Neuroendocrine tumours (NET) are a heterogeneous group of neoplasms of diffuse neuroendocrine cells. Surgery is the main aim in the treatment of NETs, which becomes impossible in the case of large tumours or infiltration into other tissues and/or important blood vessels. Neoadjuvant therapy might be helpful in decreasing NET size also, leading us to the point where a tumour, previously considered inoperable, becomes operable. The aim of the study was to assess the usage of peptide receptor radionuclide therapy (PRRT) as a neoadjuvant treatment, enabling surgical intervention in primary inoperable NET. Methods Among 47 patients treated with PRRT, 6 patients

\footnotetext{
A. Sowa-Staszczak · D. Pach · M. Trofimiuk · A. Stefańska M. Tomaszuk $\cdot$ M. Kołodziej $\cdot$ A. Hubalewska-Dydejczyk Nuclear Medicine Unit Endocrinology Department, Jagiellonian University, Medical College,
} Cracow, Poland

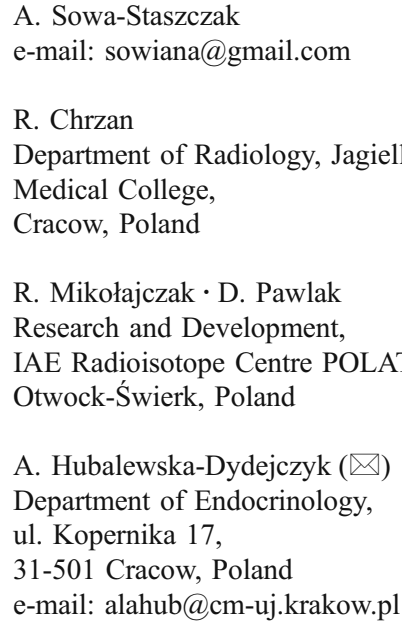

were chosen with large, inoperable tumours, for whom enabling of complete surgical excision of the lesions might offer the prospect for a cure. Response to the therapy was assessed according to Response Evaluation Criteria In Solid Tumors (RECIST).

Results The mean tumour size decreased from 6.9 (min. $3.1 \mathrm{~cm}$, $\max .9 .6 \mathrm{~cm}$ ) before therapy to $5.4 \mathrm{~cm}$ (min. $3.1 \mathrm{~cm}$, max. $9.5 \mathrm{~cm}$ ) after the treatment. According to RECIST, stabilization of the disease was observed in four and partial responses in two patients. In two patients, reduction of the tumour size enabled surgical intervention. Conclusion (1) PRRT might be considered a neoadjuvant therapy in primary inoperable NETs. (2) According to RECIST, stabilization of the disease was observed in the majority of patients. (3) We suggest that not only tumour diameter changes, but also tumour volume and contrast enhancement changes in computed tomography should be taken into consideration in assessment of the response to the therapy. (4) Somatostatin receptor scintigraphy is an important tool for qualification of the radioisotope therapy and also for the assessment of the response to PRRT.

Keywords Neuroendocrine tumours · Peptide receptor radionuclide therapy $\cdot$ Neoadjuvant therapy

\section{Introduction}

Neuroendocrine tumours (NET) are a rare, potentially malignant, usually slow-growing, mostly welldifferentiated, heterogeneous group of neoplasms of neuroendocrine cells, characterized by their ability to secrete hormones [1]. Surgical eradication is the main aim in the 
treatment of NETs, which becomes impossible in the case of large tumours or infiltration into other tissues and/or important blood vessels. Palliative surgical intervention is performed to diminish clinical symptoms and improve the patient's quality of life $[2,3]$. Another method of therapy used in patients with NETs is chemotherapy. Combined chemotherapy (e.g. cisplatin with etoposide) following incomplete surgical eradication might be considered also in patients with disseminated disease. But this kind of treatment has rather a low efficacy. To decrease tumour size, change it from untreatable to removable and make the surgical intervention possible and more effective, neoadjuvant therapy is performed on oncological illnesses (e.g. breast cancer) [4]. Neoadjuvant therapy might be helpful in decreasing NET size also, leading us to the point where a tumour, previously considered inoperable, becomes operable. There are few data on substances such as somatostatin analogues and interferon or on chemotherapy with cisplatin and etoposide used as neoadjuvant therapy. There are ongoing clinical trials of some novel targeted therapies [5]. Based on the fact that neuroendocrine neoplasms usually express somatostatin receptors, peptide receptor radionuclide therapy (PRRT) can also be used to decrease the size of the tumour and make surgical intervention possible [3]. The aim of the study was to assess the usage of PRRT as a neoadjuvant treatment leading to a decrease in the size of the tumour and enabling surgical intervention in primary inoperable NETs.

\section{Materials and methods}

Among the group of 47 patients treated with PRRT, 6 patients were chosen with large, inoperable tumours, for whom enabling of complete surgical excision of the lesions might offer the prospect for a cure. Only one of those patients had potentially removable liver metastases. All of the patients included in the therapy had positive somatostatin receptor scintigraphy (SRS) scans obtained with the use of ${ }^{99 \mathrm{~m}} \mathrm{Tc}-\mathrm{EDDA} / \mathrm{HYNIC}-\mathrm{TOC}$, and every patient was in good general condition (Karnofsky index $>70-100 \%$ ). There were three men and three women. The mean age was 58.6 years ( \pm 12.0 years, min. 46.0 years, max. 78.0 years). Among the patients included in the therapy with ${ }^{90} \mathrm{Y}$ DOTA-TATE due to inoperable NET, there were five patients with foregut tumours, among them three with pancreatic NETs and one with midgut tumour. The patient with midgut tumour presented at the time of diagnosis with diarrhoea and weight loss. The mean tumour size before therapy was $6.9 \mathrm{~cm}$ (min. $3.1 \mathrm{~cm}, \max .9 .6 \mathrm{~cm}$ ). Cytoreduction chemotherapy or long-acting somatostatin analogue were not used before PRRT. Only one patient received chemotherapy prior to PRRT, but there was no response observed in tumour size after that treatment.
Methods

Every patient received a dose of $7.4 \mathrm{GBq} / \mathrm{m}^{2}{ }^{90}$ Y-DOTATATE in 4-5 cycles every 6-9 weeks. The mean dose was 13.6 GBq (min. 11.1 GBq, max. 14.8 GBq). Response to the therapy was assessed according to Response Evaluation Criteria In Solid Tumors (RECIST).

SRS with ${ }^{99} \mathrm{mTc}-E D D A / H Y N I C-T O C$ was performed before and 1, 6 and 12 months after PRRT. The tracer used was produced fulfilling GMP criteria (POLATOM, Świerk, Poland). The diagnostic procedure was carried out in the Nuclear Medicine Unit of the Endocrinology Department, Cracow University Hospital. The first vial (containing stannous chloride, mannitol, tricine and EDDA) were completed with $1 \mathrm{ml}$ of water for injection. Then $0.5 \mathrm{ml}$ from the first vial was added to the second vial containing HYNIC-TOC. Next the tracer was labelled with 1-2 ml of ${ }^{99} \mathrm{Mo} /{ }^{99 \mathrm{~m}} \mathrm{Tc}$ generator eluate $(1-1.5 \mathrm{GBq})$ followed by $20 \mathrm{~min}$ incubation at $80^{\circ} \mathrm{C}$. The detailed method of kit labelling with ${ }^{99 \mathrm{~m}} \mathrm{Tc}$ has been presented earlier [6].

A dual-head, large field of view Proven Excellence e. cam variable angle gamma camera (Siemens) with parallel, low-energy, high-resolution (LEHR) collimators was used. After injection of the tracer $(740 \mathrm{MBq})$, all patients underwent whole-body scans after 1,4 and 24 h $(256 \times 1,024$ matrix, $12 \mathrm{~cm} / \mathrm{min}, 8 \mathrm{~cm} / \mathrm{min}, 6 \mathrm{~cm} / \mathrm{min}$, respectively) and single photon emission computed tomography (SPECT) of the abdomen and/or thorax ( $180^{\circ}$ orbit for each head, step-and-shoot mode, 64 images, at $30 \mathrm{~s}$ per view, $128 \times 128$ matrix). The acquired data were reconstructed using iterative reconstruction ordered subset expectation maximization (OSEM) 3-D FLASH (8 subsets and 10 iterations).

Before and 1-3, 6 and 12 months after the treatment, CT examinations were performed in the First CT Unit of the Radiology Department using a spiral multirow Siemens Somatom Sensation 16 CT scanner and standard protocols: (1) for abdomen and pelvis (detector configuration $16 \times$ $0.75 \mathrm{~mm}$, slice thickness and reconstruction increment 0.2 $\mathrm{cm}$, reconstruction kernel B31f, before and multiphase after i.v. nonionic contrast media administration in a dose of 1 $\mathrm{ml} / \mathrm{kg}$, flow $2.5 \mathrm{ml} / \mathrm{s}$, delay of arterial phase $30 \mathrm{~s}$ and venous phase $60 \mathrm{~s}$ after the start of contrast administration); (2) for chest (detector configuration $16 \times 0.75 \mathrm{~mm}$, slice thickness and reconstruction increment $2 \mathrm{~mm}$, reconstruction kernel B41f, before and after i.v. nonionic contrast media administration in a dose of $1 \mathrm{ml} / \mathrm{kg}$, flow $2.5 \mathrm{ml} / \mathrm{s}$, delay $30 \mathrm{~s}$ after the start of contrast administration).

Additional linear measurements of focal lesions were performed on a Siemens Wizard workstation using the tools of CT images' browser. Volume measurements of focal lesions were performed on the Siemens Wizard workstation using the dedicated volume application. For every lesion, 
its borders were manually marked on consecutive slices of the CT examination. The measurement included pixels with CT attenuation values from -30 to $200 \mathrm{HU}$ to eliminate lesions such as calcifications which influence the attenuation. Then, the program automatically calculated the volume and average attenuation of the lesion.

Chromogranin A (CGA) level, a nonspecific marker of NETs, was measured prior to and 6 months after PRRT in all patients [7].

\section{Results}

The mean tumour size decreased from $6.9(\min .3 .1 \mathrm{~cm}$, $\max .9 .6 \mathrm{~cm})$ before therapy to $5.4 \mathrm{~cm}(\min .3 .1 \mathrm{~cm}$, max. $9.5 \mathrm{~cm}$ ) after the treatment. According to RECIST, stabilization of the disease was observed in four and partial responses in two patients. In the case of patient 6 (Table 1, Fig. 1), the tumour size 1 month after PRRT decreased from 11.0 (before the treatment assessed on X-ray photos) to 7.2 $\mathrm{cm}$ (the longest diameter of lesions on axial CT slices). Five months later, a further decline in the tumour size to $5.7 \mathrm{~cm}$ was observed. Reduction of the tumour size after neoadjuvant PRRT enabled qualification for a laparotomy, which was performed 11 months after PRRT. Due to infiltration of the large vessels, which was visible on CT

Table 1 Quantitative evaluation in CT of diameter, volume and attenuation of tumour before and 6 months after PRRT

\begin{tabular}{|c|c|c|c|c|c|c|}
\hline Patient & 1 & 2 & 3 & 4 & 5 & 6 \\
\hline Sex & $\mathrm{F}$ & M & M & M & $\mathrm{F}$ & M \\
\hline Age & 78 & 56 & 61 & 70 & 59 & 48 \\
\hline $\begin{array}{l}\text { D prior to PRRT } \\
(\mathrm{cm})\end{array}$ & 3.4 & 7.9 & 3.1 & 9.6 & 5.8 & 7.2 \\
\hline $\begin{array}{l}\text { V prior to PRRT } \\
\left(\mathrm{cm}^{3}\right)\end{array}$ & 19.9 & 230.9 & 3.8 & 301.5 & 75.8 & 208.3 \\
\hline $\begin{array}{l}\text { A prior to PRRT } \\
(\mathrm{HU})\end{array}$ & 56.8 & 28.5 & 46.7 & 70.8 & 68 & 44.9 \\
\hline $\begin{array}{l}\text { D after PRRT } \\
(\mathrm{cm})\end{array}$ & 3.4 & 4.2 & 3.1 & 9.5 & 6.5 & 5.7 \\
\hline $\begin{array}{l}\text { V after PRRT } \\
\left(\mathrm{cm}^{3}\right)\end{array}$ & 19.8 & 44.7 & 3.7 & 336.2 & 84.0 & 82.4 \\
\hline $\begin{array}{l}\text { A after PRRT } \\
(\mathrm{HU})\end{array}$ & 62.9 & 29.5 & 42.5 & 53.2 & 59.8 & 40.5 \\
\hline $\begin{array}{l}\text { Response to the } \\
\text { therapy }^{\mathrm{a}}\end{array}$ & SD & PR & SD & SD & SD & PR \\
\hline D change $(\%)$ & 0 & -47.8 & 0 & -1.0 & 12.1 & -20.7 \\
\hline $\mathrm{V}$ change $(\%)$ & -0.6 & -80.7 & -1.1 & 11.5 & 10.9 & -60.7 \\
\hline A change $(\%)$ & 10.7 & 3.5 & 9.0 & -24.9 & -12.1 & -9.8 \\
\hline
\end{tabular}

$\overline{D \text { diameter, } V \text { volume, } A \text { attenuation, } S D \text { stabilization of the disease, }}$ $P R$ partial response

${ }^{\text {a }}$ Response to the therapy according to RECIST, which includes measurement of the longest diameter of every measurable lesion on axial CT slices and calculation of the sum of the longest diameters for all selected target lesions scans, only partial removal of the tumour was possible. The patient qualified for additional PRRT courses. An additional three PRRT courses were given and further resection of the tumour size (from 5.7 after surgery to $3.6 \mathrm{~cm}$ ) was considered, but the tumour remains still inoperable.

The second patient (patient 2 in Table 1, Fig. 2) with partial response qualified for surgery. After the PRRT, the tumour size decreased from 7.9 to $4.2 \mathrm{~cm}$ and there was no evidence of the liver metastases observed prior to PRRT. Whether complete tumour removal can be achieved is an open question.

The mean time of observation was 21.5 months ( \pm 15.3 , min. 6.0 months, max. 46.0 months). The median CGA decreased from 33.4 (min. $12.8 \mathrm{U} / 1$, max. $268.7 \mathrm{U} / \mathrm{l}$ ) to $27.15 \mathrm{U} / 1$ (min. $21.8 \mathrm{U} / 1$, max. $36.3 \mathrm{U} / \mathrm{l}$ ). No correlation between tumour size and uptake intensity and CGA was observed. PRRT did not cause myelotoxicity or nephrotoxicity.

\section{Discussion}

Neoadjuvant therapy is a kind of induction therapy leading to shrinkage of the tumour, which enables surgical intervention. It is widely used in different types of cancers (e.g. breast cancer) and may include chemotherapy, radiotherapy and/or hormone therapy [4]. There are also possibilities for neoadjuvant therapy in the case of NETs, as mentioned above. PRRT might be also considered as a neoadjuvant therapy in the case of NETs $[3,8]$.

Expression of somatostatin receptors on the surface of NETs became the molecular basis for the use of somatostatin analogues in the diagnosis and therapy of these neoplasms. PRRT with the use of labelled somatostatin analogues has been applied in disseminated and inoperable NETs with positive somatostatin receptor expression confirmed by SRS.

The results of our study indicate, as also presented by Kaemmerer et al., that PRRT might be considered not only as a palliative but also as a neoadjuvant therapy in primary inoperable NETs [3]. In the case presented by Kaemmerer et al., PRRT and the following surgical intervention led to complete remission of the disease. In another case described recently by Stoeltzing et al. after surgical removal of the large primary tumour of the pancreatic tail ${ }^{90} \mathrm{Y}$ DOTATOC was used with neoadjuvant intention resulting in a regression of hepatic lesions and facilitating surgical removal of all remaining liver metastases in a second operation [8]. In our study we observed reduction in the tumour size/volume in two of the patients with primary inoperable tumours. In one case (patient 6 in Table 1), PRRT enabled surgical intervention, but complete resection of the tumour was not possible due to infiltration of the 


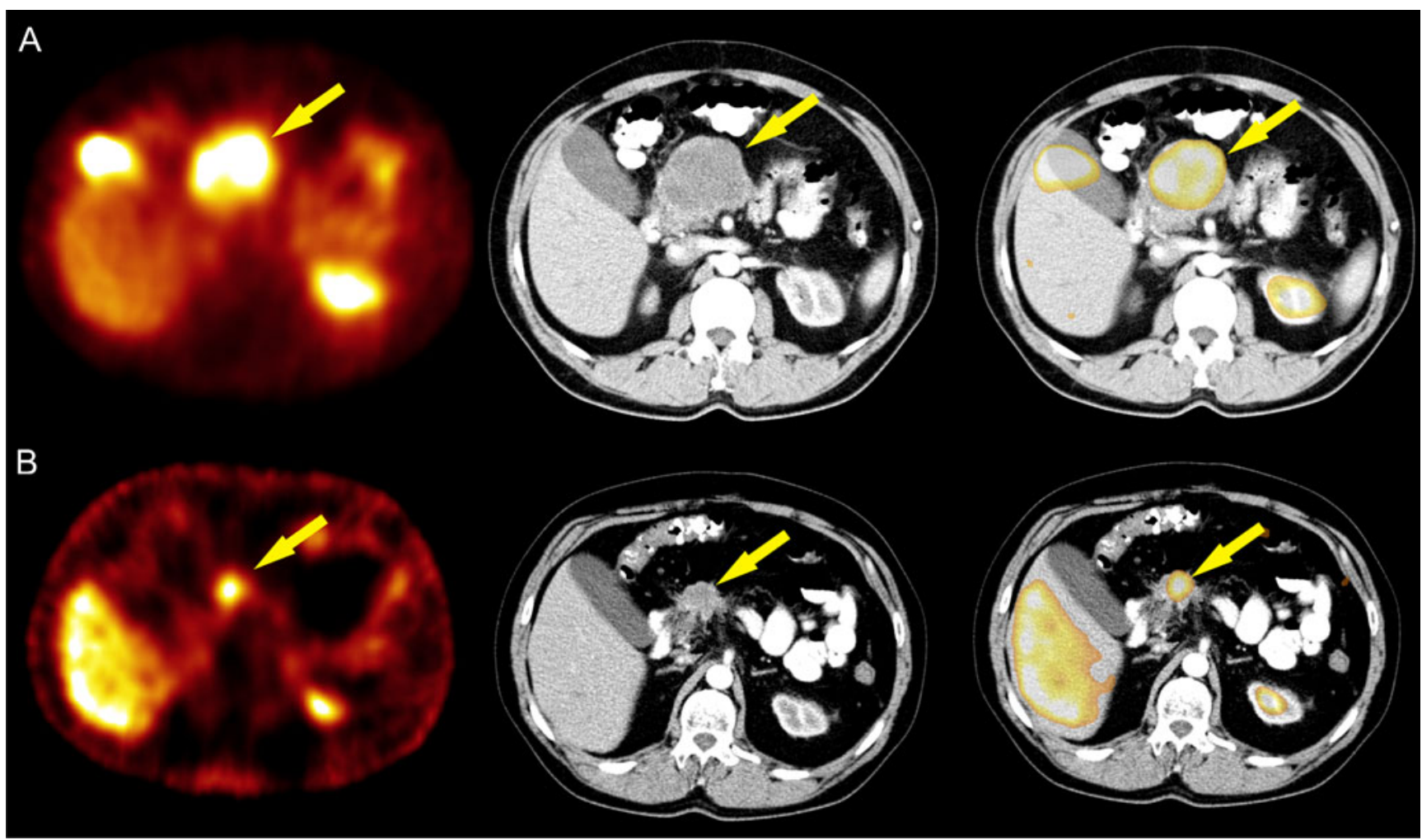

Fig. 1 A 48-year-old man with inoperable midgut tumour (patent 6). The results of ${ }^{99 m}$ Tc-EDDA/HYNIC-TOC SRS, CT and SRS/CT fusion image before (a) and after (b) the neoadjuvant therapy with PRRT are presented

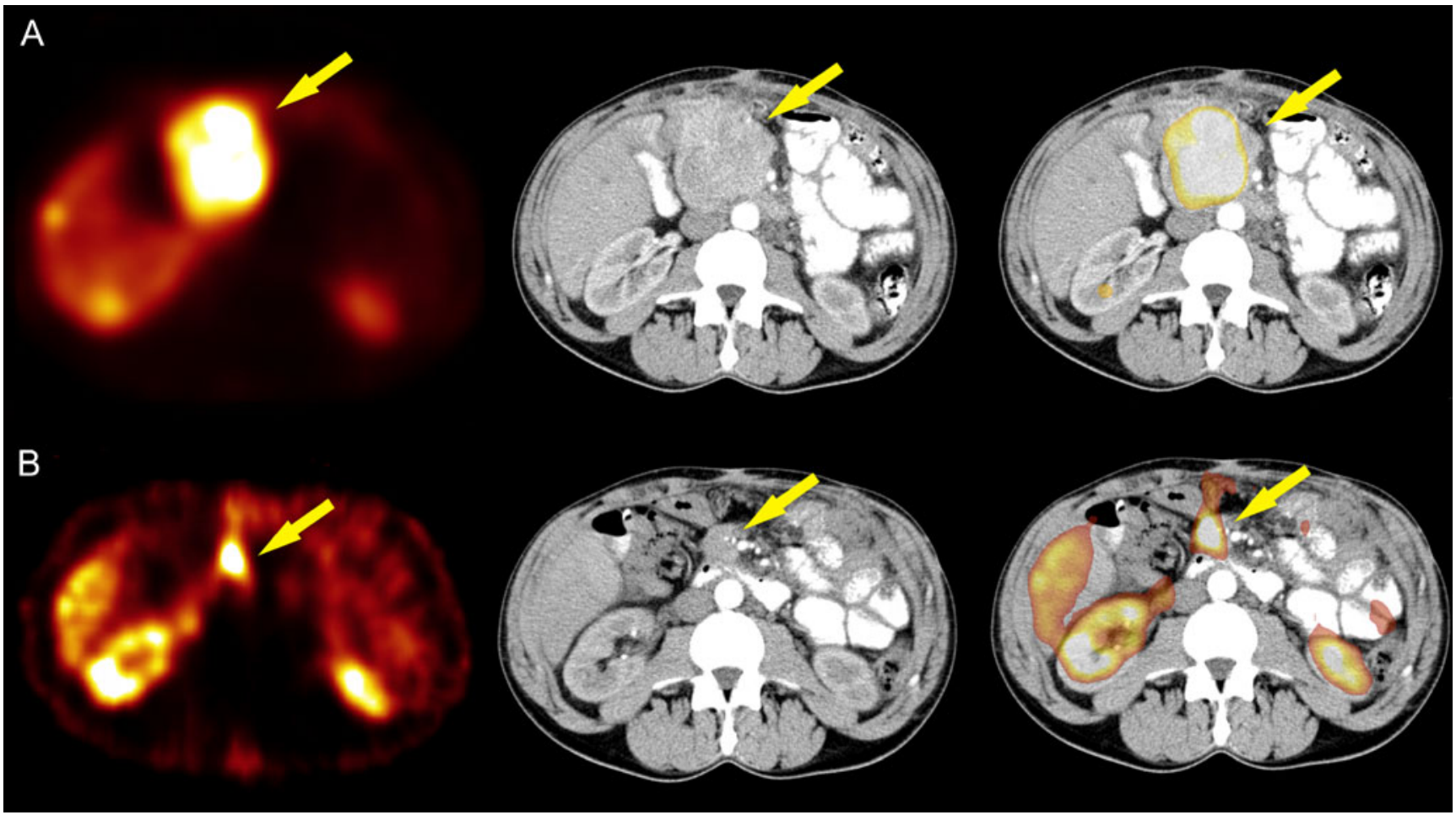

Fig. 2 A 56-year-old man with inoperable pancreatic NET (patient 2). The results of ${ }^{99 \mathrm{~m}} \mathrm{Tc}-\mathrm{EDDA} / \mathrm{HYNIC}-\mathrm{TOC}$ SRS, CT and SRS/CT fusion image before (a) and after (b) the neoadjuvant therapy with PRRT are presented 
vessels. This patient underwent a second PRRT cycle, which led to further reduction in the tumour size. The second patient (patient 2 in Table 1) with partial response has qualified for surgery. This patient presented with liver metastases prior to PRRT, and there was no evidence of hepatic lesions after radioisotope therapy. These results show the potential role of PRRT as a neoadjuvant treatment. Moreover, the use of PRRT as neoadjuvant therapy does not have to be limited to patients with NETs with inoperable primary tumour without dissemination, but as suggested by Stoeltzing et al. and what was also seen in the case of one of the patients treated in our centre, might be considered also in patients with liver metastases [8].

We also analysed the results of the CT scans performed prior to and after the treatment. The assessment according to RECIST $[9,10]$ includes measurement of the longest diameter of every measurable lesion on axial CT slices and calculation of the sum of the longest diameters (SLD) for all selected target lesions.

For partial response (PR), at least a $30 \%$ decrease in the SLD of target lesions should be found, taking the baseline SLD as reference. However, it should be remembered that the volume changes proportionally to the cube of the linear measurement, so assessment of the linear diameter change may only insufficiently estimate the volume difference in the course of treatment. Another inconvenience of the RECIST standard is that it takes into consideration only the size of lesions, with no assessment of structural changes in the course of treatment, manifesting on $\mathrm{CT}$ as changes of $\mathrm{X}$-ray attenuation values (reduction of attenuation, weaker contrast enhancement as the effect of neoplastic tissue necrosis). In one of the cases analysed in this paper (patient 4 in Table 1), in spite of no significant tumour diameter reduction (decrease of linear diameter by about $1 \%$ ), an average lesion attenuation decreased by about $24.9 \%$, probably as the effect of therapy. In another case (patient 6 in Table 1), there was no possibility to assess a CT scan performed prior to the PRRT (only an X-ray photo version from 2005 was available). Therefore, the CT scan 1 month after PRRT was compared with a scan performed 6 months after radioisotope therapy. In the $\mathrm{CT}$ scan before the treatment, the size of the tumour was $11.0 \mathrm{~cm}$. In our analysis 1 and 6 months after the treatment, we observed a significant decrease in neoplastic tissue volume of about $60.7 \%$, but the SLD decrease was only $20.7 \%$. In another group of patients with NETs, treated in our department with ${ }^{90}$ Y-DOTA-TATE and/or ${ }^{177}$ Lu-DOTA-TATE, we also observed a similar discrepancy with significant decrease in lesion volume and only slight SLD decrease, assessed as stabilization of the disease according to RECIST. In some neoplasms characterized by good vascularization, modified standards of therapy response are used more and more commonly in $\mathrm{CT}$, taking into consideration attenuation changes, for example the Choi criteria for gastrointestinal stromal tumour (GIST) and modified RECIST for hepatocellular cancer [11, 12]. Because NET lesions are mostly well-vascularized neoplasms, a rational solution would be the drawing up of CT assessment criteria for such lesions, taking into consideration attenuation and contrast enhancement changes in the course of treatment.

We would like to emphasize also a role of scintigraphy in evaluating disease and in assessment of the response to the therapy. Scintigraphy is an important tool for qualification of the radioisotope therapy and also for the assessment of the response to PRRT. The best option is to assess both scintigraphy and CT together, which may give more information about staging of the disease.

\section{Conclusion}

1. PRRT might be considered a neoadjuvant therapy in primary inoperable NETs.

2. According to RECIST stabilization of the disease was observed in the majority of patients. In two patients, reduction of the tumour size enabled surgical intervention.

3. We suggest that not only tumour diameter changes, but also tumour volume and contrast enhancement changes on $\mathrm{CT}$ should be taken into consideration in assessment of the response to the therapy.

4. SRS is an important tool for qualification of the radioisotope therapy and also for the assessment of the response to PRRT.

Open Access This article is distributed under the terms of the Creative Commons Attribution Noncommercial License which permits any noncommercial use, distribution, and reproduction in any medium, provided the original author(s) and source are credited.

\section{References}

1. Modlin IM, Oberg K, Chung DC, Jensen RT, de Herder WW, Thakker RV, et al. Gastroenteropancreatic neuroendocrine tumours. Lancet Oncol 2008;9:61-72. PMID 18780869.

2. Oberg K, Kvols L, Caplin M, Delle Fave G, de Herder W, Rindi G, et al. Consensus report on the use of somatostatin analogs for the management of neuroendocrine tumors of the gastroenteropancreatic system. Ann Oncol 2004;15:966-73. PMID 15151956.

3. Kaemmerer D, Prasad V, Daffner W, Hörsch D, Klöppel G, Hommann $\mathrm{M}$, et al. Neoadjuvant peptide receptor radionuclide therapy for an inoperable neuroendocrine pancreatic tumor. World J Gastroenterol 2009;15:5867-70. PMID 19998512.

4. Shimizu T, Hirano A, Watanabe O, Kinoshita J, Kimura K, Kamimura M, et al. Successful neoadjuvant therapy with trastuzumab, paclitaxel and epirubicin for an elderly patient with inflammatory breast cancer. Anticancer Res 2010;30:581-5. PMID 20332474. 
5. Gabriel M, Andergassen U, Putzer D, Kroiss A, Waitz D, Von Guggenberg E, et al. Individualized peptide-related-radionuclidetherapy concept using different radiolabelled somatostatin analogs in advanced cancer patients. Q J Nucl Med Mol Imaging 2010;54:92-9. PMID 20168291.

6. Plachcinska A, Mikolajczak R, Maecke HR, Mlodkowska E, Kunert-Radek J, Michalski A, et al. Clinical usefulness of 99mTcEDDA/HYNIC-TOC scintigraphy in oncological diagnostics: a pilot study. Cancer Biother Radiopharm 2004;19:261-70. PMID 15186607.

7. Modlin IM, Gustafsson BI, Moss SF, Pavel M, Tsolakis AV, Kidd M. Chromogranin A-biological function and clinical utility in neuro endocrine tumor disease. Ann Surg Oncol 2010;17: 2427-43.

8. Stoeltzing O, Loss M, Huber E, Gross V, Eilles C, Mueller-Brand $\mathrm{J}$, et al. Staged surgery with neoadjuvant 90Y-DOTATOC therapy for down-sizing synchronous bilobular hepatic metastases from a neuroendocrine pancreatic tumor. Langenbecks Arch Surg 2010;395:185-92. PMID 19506898.
9. Therasse P, Arbuck S, Eisenhauer E, Wanders J, Kaplan RS, Rubinstein L, et al. New guidelines to evaluate the response to treatment in solid tumors. European Organization for Research and Treatment of Cancer, National Cancer Institute of the United States, National Cancer Institute of Canada. J Natl Cancer Inst 2000;92:205-16. PMID 10655437.

10. Eisenhauer E, Therasse P, Bogaerts J, Schwartz LH, Sargent D, Ford R, et al. New response evaluation criteria in solid tumours: revised RECIST guideline (version 1.1). Eur J Cancer 2009;45:228-47. PMID 19097774.

11. Choi H, Charnsangavei C, Faria SC, Macapinlac HA, Burgess MA, Patel SR, et al. Correlation of computed tomography and positron emission tomography in patients with metastatic gastrointestinal stromal tumor treated at a single institution with imatinib mesylate: proposal of new computed tomography response criteria. J Clin Oncol 2007;25:1753-9. PMID 17470865.

12. Llovet J, Di Bisceglie A, Bruix J, Kramer BS, Lencioni R, Zhu AX, et al. Design and endpoints of clinical trials in hepatocellular carcinoma. J Natl Cancer Inst 2008;100:698-710. PMID 18477802. 